\title{
Brain Arteriovenous Malformations Classifications: A Surgical Point of View
}

\author{
Giovanni Marco Sicuri, Nicola Galante, \\ and Roberto Stefini
}

\section{Introduction}

Brain arteriovenous malformations are complex developmental malformations that primarily affect the leptomeningeal vessels. AVMs are subject to a dynamic process that leads to dilation of arteries and veins and to the pathological modification of their elastic and muscular laminae. Involvement of perforating vessels may add damage of the adjacent neural tissue. In typical plexiform AVMs, the nidus of the malformation is composed of a network of abnormal vessels interposed between the feeding arteries and the draining veins [1].

Hemorrhage is the most frequent onset symptom, although AVM can also occur with epileptic seizures, progressive focal neurological deficits, and neurocognitive deficits. Surgery is still considered the most effective and definitive treatment; endovascular occlusion and radiosurgery can be considered as alternatives in specific cases. However, each treatment might carry potential transient or permanent postoperative sequelae. Patient selection should therefore weigh the natural history of AVMs against treatment-related risks. It is a difficult process complicated by the variety of angioarchitecture, size, location, and clinical features of AVMs. It is, however, the key to achieving a good outcome.

A good classification system should assess therapeutic risks, surgical challenges, and operative morbidity. After validation, its first purpose is to guide the physician to present to the patient the optimal treatment option. At the same time, it may facilitate communication among researchers on the best care. An ideal scheme should be assessed at bedside and contemplate few variables; otherwise it may be cumbersome. However, even oversimplification has some drawbacks and does not exclude the risk of over-grading or

G. M. Sicuri · N. Galante $\cdot$ R. Stefini $(\varangle)$

Department of Neurosurgery, ASST Ovest Milanese, Legnano, Italy

e-mail: giovannimarco.sicuri@asst-ovestmi.it;

nicola.galante@asst-ovestmi.it; roberto.stefini@asst-ovestmi.it under-grading for the interobserver variability. For instance, it is common experience that neuroradiologists tend to undergrade, whereas the neurosurgeons tend to over-grade a brain AVM classified by Spetzler-Martin (S-M) grading [2]. Furthermore, agreement on the overall grade differed depending on imaging modalities; the best agreement came from using Computer Tomographic Angiography and Magnetic Resonance Imaging rather than Digital Subtraction Angiography [3].

\section{Classification Schemes on AVM Operability}

The first and most common classification systems are those that assess AVMs' operability by balancing the risks of surgical treatment (Fig. 1). These schemes are based on predictive factors extrapolated from the neuroradiological exams and the clinical features of the patient. The most important anatomical factors appear to be the size and the location of the AVM's nidus. An arterial supply prevalent from perforating arteries and the location in an eloquent area can complicate surgery. A deep venous drainage is used as an indicator of deep-seated extension of AVMs and of the presence of small choroidal vessels around the vein in the white matter that can cause some technical difficulties and promote postoperative focal neurological deficit [4-7].

In addition, the more compact the AVM's nidus, the easier the surgical resection. Hemodynamic predictive factors have been related to the onset of hyperemic complications, represented by the formation of vasogenic edema or bleeding in the territories surrounding the AVM after its removal. The onset of these events is related to a venous hyperemia resulting from the occlusion of venous drainage and an arterial hyperemia related to the reperfusion of previously hypoperfused brain areas due to blood steal phenomena. D'Aliberti et al. correlated the onset of hyperemic complications with venous times measured in angiography. The "early venous time" was defined as the time of appearance of the nidus and 
Fig. 1 Timeline of brain arteriovenous malformations classifications

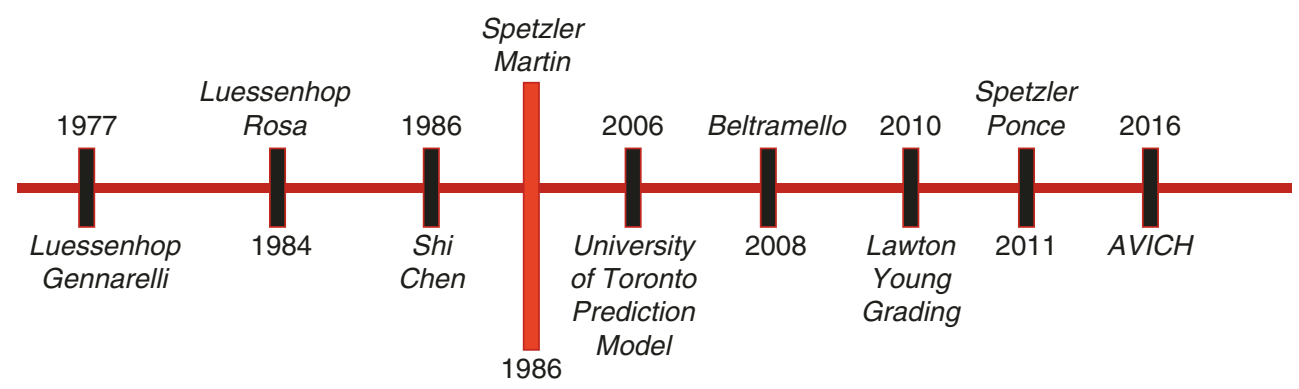

of the veins directly draining the AVM, while the "intermediate venous time" was defined as the time of appearance of the recruited veins, namely the veins draining the brain parenchyma afferent to a drainage of the AVM. These venous times were considered altered when very short or when there was a prolonged interval with the "late venous time," namely the time of appearance of normal brain veins. The altered times were closely correlated with the incidence of hyperemic complications, which accounted for about $50 \%$ of adverse events in the series [8].

Furthermore, clinical factors negatively correlated with the outcome are the advanced age, the presence of neurological deficits or an alteration of the state of consciousness at the time of treatment, and the presence of a previous bleeding [7].

\section{Pre-Spetzler-Martin (S-M) Classifications}

The first relevant classification was developed by Luessenhop and Gennarelli in 1977 [9]. Data were exclusively related to supratentorial AVM. AVMs were divided into four degrees according to the number of arterial pedicles in a single vascular territory. Lenticulostriate arteries and Heubner's recurrent artery were considered separate feeders. The greater the number of peduncles involved, the greater the difficulty of resection. Grade IV AVMs were considered inoperable. The presence of subarachnoid hemorrhage was managed as an unfavorable factor, and an eloquent area was considered relevant for morbidity. In 1984, Luessenhop and Rosa simplified the grading of the previous classification [10]. Assuming that the size of the AVM's nidus is related to the number of arterial pedicles, the grade was defined according to the diameter of the nidus: $>2 \mathrm{~cm}$ was grade I, $2-4 \mathrm{~cm}$ grade II, 4-6 cm grade III, and $>6 \mathrm{~cm}$ grade IV. Cerebellar AVMs were included. Surgical results indicated that in grade I and II the mortality and morbidity of surgical treatment were probably lower than the natural risk of the disease. In 1986 Shi and Chen presented an alternative classification [11]. More factors were included in the classification: dimension, location, complexity of arterial supply, and venous drainage. Each of the four factors was subclassified in grade I-
IV. When all the criteria matched, the AVM was of that grade. When only one of the criteria was of a higher degree, the AVM was of intermediate grade. However, when at least two criteria were of the same highest grade, the AVM would have been of the latter degree. Therefore, three intermediate degrees were possible in addition to the four main degrees. The authors demonstrated an increased risk with increasing AVM grade with a $20 \%$ mortality in grade III-IV AVMs.

\section{Spetzler-Martin Classification and Following Edits}

In 1986, Spetzler and Martin published a new classification with the aim of simplifying the analysis and reducing the variables while maintaining a good predictive value [12]. The basis for simplification was the concept that many of the predictive factors are interrelated. This scheme has become the predominant pattern in the classification of cerebral AVMs. The only criteria included in scoring were the nidus size, the venous drainage pattern, and the AVM location. Each factor had an independent score that was added to the others. The dimensions of the nidus were separated into three grades: when $<3 \mathrm{~cm}$, one point was assigned; if between 3 to $6 \mathrm{~cm}$, two points; over $6 \mathrm{~cm}$, three points. The authors implied that the greater the size of the nidus, the greater the brain tissue exposed at risk during surgery. Size was also directly related to the number of vascular afferent peduncles, to the flow and to the degree of blood steal. When the venous drainage pattern included only the cortical veins, it was regarded as superficial, and no points were therefore assigned. Conversely, when it involved the deep venous system (internal cerebral veins, basal veins, precentral cerebellar vein), one point was assigned. A deep venous drainage was related to the surgical accessibility to the AVM because deep veins are difficult to coagulate and can retract into the adjacent cerebral parenchyma and into the ventricle. Lastly, one point was assigned to AVMs located in eloquent areas. Elective areas were considered the sensory motor cortex, the area of language, the visual cortex, the hypothalamus and the thalamus, the internal capsule, the brain stem, the cerebellar peduncles, and the deep cerebellar nuclei. 
The grade of the AVM was derived from the sum of the scores assigned for each category. Therefore, AVMs of grade I-V were identified. The simplicity of application of this classification system certainly contributed to its diffusion. Moreover, a low number of variables increased the statistical power of scientific studies, enlarging the number of subjects assigned to each category. Some drawbacks, however, have been identified: The compactness of the nidus and the involvement of deep arterial afferents, although important to define the surgical complexity, were not considered. Furthermore, the definition of eloquence of a brain area cannot be always accurate. It is known that, especially for higher-level cognitive functions, structural anatomy often does not fully correspond to functional anatomy. Eventually, S-M grading did not take into account the patient's clinical and neurological status.

The weakest point of this classification consisted in its lower power to define an accurate outcome in Grade III patients. Indeed, grade III AVMs were not a homogeneous group, with varying risks of morbidity and mortality. For these reasons, in 1998 de Oliveira et al tried to subclass the grade III AVMs by defining a grade IIIa and a grade IIIb [13]. In grade IIIa, the score was given by the large size of the nidus $(>6 \mathrm{~cm})$, while in grade IIIb the score was determined by the deep drainage and/or the eloquent area. The authors advised against surgical treatment for grade IIIb AVMs, albeit with some exceptions. In 2003, Lawton further subdivided grade three AVMs into four subtypes [14]. According to his classification, AVMs $<3 \mathrm{~cm}$ with deep drainage and located in an eloquent area (grade III-) had a low surgical risk, similar to that of low-grade AVMs. The 3-6 cm AVMs with deep drainage (plain grade III) had an intermediate risk, while the 3-6 cm AVMs in an eloquent area had a high surgical risk, similar to that of high-grade AVM. AVMs $>6 \mathrm{~cm}$ with superficial drainage in a non-eloquent area were absent in the surgical series described (grade III *). The authors recommended surgical resection in Grade III and III-AVMs.

Since experience showed that the AVMs of Grade I and II and IV and V of the score of Spetzler and Martin perform similarly, in 2011 Spetzler and Ponce condensed the original classification into only three degrees [15]. Grade I and II AVMs were merged into grade A, Grade III AVMs were assigned grade B, Grade IV and V grades were condensed into grade $\mathrm{C}$. The predictive power on surgical outcome was identical to the original classification. The authors generally recommended surgical treatment for grade A AVMs, multimodal treatment for grade $\mathrm{B}$, and observation for grade $\mathrm{C}$, reserving treatment for cases of recurrent hemorrhages or progressive neurological deficits due to blood steal phenomena. This scheme offered the advantage of increased samples for statistical analysis, but it did not dissolve the lack of homogeneity of Grade III AVMs.
Two alternative schemes were developed after the Spetzler-Martin classification: the University of Toronto Prediction Model and the Lawton-Young Grading System. Both contested the excessive simplicity of the classification of Spetzler-Martin in an effort to increase the discriminative power on the outcome of AVM patients after surgery.

The former model was published in 2006 after a retrospective analysis of 175 patients [16]. The authors added nidus morphology to significant predictors of short- and long-term neurological outcomes in addition to the eloquent area and deep venous drainage. A diffuse nidus morphology was defined as a nidus including normal brain parenchyma, with no defined margins, as opposed to a compact nidus. On the other side, the size of the nidus $>3 \mathrm{~cm}$, the deep arterial supply, and the presence of associated aneurysms were not significant in the retrospective analysis and were excluded from the model. The model assigned a relative weight to the three factors considered: the eloquent area was assigned four points, the diffusivity of the nidus three points, the deep venous drainage two points. Low-risk patients ( $0-2$ points), medium-risk (3-5 points) and high-risk patients (6-7 points) were stratified. The authors showed in the series that the proposed scheme had a superior discriminative power with respect to the grading of S-M in predicting permanent neurological deficits, especially in low-grade AVMs.

In 2010 Lawton and Young analyzed 300 patients undergoing AVM surgical resection and developed a new grading system [17]. Three variables were included in the scheme: age, hemorrhagic presentation, and nidus compactness. Similarly to the S-M scale the scoring ranged from one to five points: one point was assigned for age $<20$ years, two points for 20-40 years, three points for $>40$ years; one point was assigned for AVMs with diffuse nidus and one point for hemorrhagic presentation. The predictive accuracy of the neurological outcome of the proposed model was higher than the S-M scale and was even higher by adding the scores obtained with the S-M grading and their grading [18]. Lawton et al. concluded that the risk of a surgical resection was acceptable in patients with grades $\leq 3$ according to the Lawton-Young grading system and grades $\leq 6$ according to the score obtained from the sum of the their grading and S-M scale. Like the Toronto Prediction Model, the study did not confirm a predictive value of deep arterial support.

\section{Other Operative AVM Classification Schemes}

Classifications described above are useful for defining the risk of microsurgical resection of an AVM according to the clinical and angioarchitectural features. However, over the past few decades, alternative or combined treatments have been developed and refined. Radiosurgery and endovascular 
surgery are effective on AVM exclusion by the administration of ionizing radiations and by the obliteration with embolic liquids, respectively. Since the techniques mentioned obviously use methods other than microsurgery, risk assessment criteria used for surgical treatment cannot be as valid. For both techniques, specific classifications for predicting the complication rate and treatment success were developed over time [19-26].

All these schemes have shown some limitations. First, since treatment is often multimodal, an exact definition of the risk is difficult and the comparison between the series is complex. In addition, the ARUBA study recently showed that the risk of death and stroke in unruptured AVM was higher with interventional treatment than with medical therapy. Although this study has been severely criticized for its design, conduct, analysis, and interpretation, it has brought out the importance of weighing the natural history of the disease and its most frequent and feared complication, hemorrhage, with treatment-related risks. A mere risk stratification for a type of treatment may miss that goal. Interestingly, Beltramello et al. drew up a score that considers the psychosocial aspects of the patient and that is valid for all modalities of AVM treatment [27]. The authors proposed an AVM Cumulative Score that summed the Intention to Treat Score (ITS) and the Treatment Risk Score (TRS). The former was composed of the sum of patient clinical features and morphological features of the AVM: age $(<50$ years: 0 point; 50-65 years: 1; >65 years: 2); previous bleeding (yes: 0 points; no: 2); neurological deficit unrelated to previous bleeding (yes: 0 points; no: 1); patient's firm purpose to be treated (yes: 0 point; no: 1); AVM size $<10 \mathrm{~cm}^{3}$ (yes: 0 points; no 1); deep location (yes: 0 points; no: 1); deep venous drainage only (yes: 0 points; no: 2 ); varices or associated aneurysms (yes: 0 points; no: 2 ). The ITS therefore ranged from 0 to 12 . The TRS was instead calculated differently according to the chosen treatment and ranged from 0 to 5. When a surgical treatment was indicated, the S-M scales that divides patients into five categories was used, with the difference that the grade III AVM with vascular supply from the lenticulostriate arteries marked 4 points instead of 3 . The authors based the radiosurgical risk prediction exclusively on the size of the nidus $\left(<5 \mathrm{~cm}^{3}: 1\right.$ point; $5-10 \mathrm{~cm}^{3}: 2$; $10-20 \mathrm{~cm}^{3}: 3 ; 20-30 \mathrm{~cm}^{3}: 4 ;>30 \mathrm{~cm}^{3}: 5$ ); however, they subtracted one point in low-flow AVMs, as they believed that this aspect was associated with a higher rate of obliteration in shorter time. Eventually, factors considered in the endovascular risk prediction were the AVM volume $\left(<10 \mathrm{~cm}^{3}: 1\right.$ point; $>10 \mathrm{~cm}^{3}$ : 2); the eloquent area (yes: 1 point; no: 0 ); the presence of perforating feeders (yes: 1 point; no: 0); and an unfavorable AVM angioarchitecture (yes: 1 point; no: 0). The Cumulative Score was formed by the sum of ITS and RTS. The authors recommended treatment when $\leq 10$, offered it with substantial risk if 11 or 12 , advised against it when between 13 and 17. The same group then validated this classification on 104 treated patients, with only a slight change to the points assigned to age ( $<40$ years: 0 point; 40-60 years: 1 ; >60 years: 2) [28].

All classifications described so far are useful in elective cases. In 2016 Neidert et al. proposed a new grading system to predict the outcome of patients with intracerebral hemorrhage (ICH) due to AVM rupture [29]. Scoring systems that predict outcome in spontaneous ICH may not be reliable in AVM patients because the pathophysiology of bleeding is different. Indeed, outcome in spontaneous ICH has been shown to be worse than in AVM-related ICH. Prognosis may then be more linked to the angioarchitectural features of the AVM than to the ICH itself. The proposed score included the sum of the scores obtained from factors derived from the S-M scale and the Lawton-Young grading system (size, deep drainage, eloquent area, age, compactness of the nidus) and from factors derived from the ICH Score [30], namely the GCS score (13-15: 0 points, 5-12: 1; 3-4: 2); volume of intracerebral hemorrhage $\left(<30 \mathrm{~cm}^{3}: 0\right.$ points; $\left.>30 \mathrm{~cm}^{3}: 1\right)$; presence of intraventricular hemorrhage (no: 0 points, yes: $1)$. The score therefore ranged from 2 to 13 . In a recent validation it was confirmed that with a score $\geq 9$ there was a favorable outcome in only $5.7 \%$ of cases, while in $1.4 \%$ with a score $\geq 10$ [31].

\section{Topographic AVM Classifications}

Anatomical classifications have been devised for AVM located in specific anatomical areas of the brain, highlighting some peculiarities that may be useful for the surgeon. AVMs of the sylvian fissure were classified by Sugita in 1987 [32] into four subtypes: pure sylvian AVMs, located in the subarachnoid space around the middle cerebral artery without a parenchymal base; sylvian lateral AVMs, located in the lateral part of the sylvian fissure and in the temporal lobe cortex; medial sylvian AVMs, located in the medial part of the sylvian fissure and in the frontal lobe; deep sylvian AVMs, located in the deepest part of the sylvian fissure and in the insular cortex. Lawton et al. in 2007 published an application of the Sugita classification on 28 surgical patients [33]. In both papers, the classification itself did not effect a significant change in the outcome but was useful to indicate some surgical tips. First, deep AVMs required a slight posterior extension of the craniotomy to allow a wider opening of the sylvian fissure to reach the nidus, since the trunks of the middle cerebral artery (ACM) normally lay on the surface of the nidus. In pure AVMs, branches of the ACM were instead under the nidus, while the venous drainage was superficial in the middle cerebral veins; in the latter the authors advised the 
dissection of the sylvian fissure from distal to proximal to better preserve the en passage arteries. In medial AVMs, the greatest surgical challenge was generally the coagulation of the feeding branches from lenticulostriate arteries, while in lateral AVMs it was the control of the branches of the anterior choroidal artery.

The San Francisco Group described other specific locations of the AVMs. AVMs of the midline were classified into five subtypes according to location (anterior, middle, or posterior) and depth (superficial and deep) [34]. Compared to other AVMs of the convexity, which can be approached perpendicularly, surgical excision was judged to be more complex, because dissection of the nidus along the medial side in superficial parafalcine AVMs and along all four sides in deep parafalcine AVMs must be tangential, though requiring brain retraction and mobilization of the nidus. For each subtype, the authors described a peculiar strategy of patient positioning and operating table movements, in order to help the surgeon with gravity retraction to better expose the nidus and the afferent arterial branches. The highest risk was attributed to superficial-middle AVMs, probably due to the more extensive arterial supply (greater percentage of two or three vascular territories) and the adjacency to the sensory-motor cortex.

AVMs of the temporal lobe were subdivided into five subtypes [35]. Lateral AVMs were found immediately beneath the convexity and could be approached perpendicularly with a pterional approach. The other subtypes required more complex approaches (subtemporal for basal, orbytozygomatic for medial, transylvian for sylvian and transcortical for ventricular AVMs) and tangential dissections. The classification was not found useful for predicting the outcome, described as generally good.

For cerebellar AVMs, five anatomic subtypes were identified: three corresponded to the three cerebellar surfaces described by Rhoton (suboccipital, tentorial, petrosal), to which the vermian and tonsillar AVMs were added [36]. A torcular craniotomy with transverse sinus lift was generally performed for vermian and tentorial, a retrosigmoid craniotomy for the petrosal, a medial suboccipital craniotomy for tonsillar, a lateral suboccipital craniotomy for suboccipital AVMs. The outcome was better in the tentorial and tonsillar AVMs, worse for the petrosal and vermian ones.

Brainstem AVMs were classified into six subtypes: anterior and posterior midbrain, anterior or lateral pontine and anterior or lateral medullary [37]. Lateral AVMs could generally be resected, whereas AVMs with more anterior localization, or when it was difficult to distinguish between normal and feeding perforating branches, were treated with "occlusion in situ," namely closing of the feeding arteries and of the drainage veins leaving the nidus in the brainstem. The authors recommended surgical treatment only in patients with AVMs at high risk of rupture.

\section{Conclusions}

Over the years, several classifications for AVMs have been proposed, some favoring simplification and others preferring accurateness, at the expense of a lower feasibility. So far, the Spetzler-Martin classification has been used most often to quickly assess the operability of AVMs. However, AVM treatment is increasingly multimodal and evolving, so parameters to consider are changing. Clinical and neuroradiological evaluation will in the near future be complemented by genetic and molecular factors; thus, clinical trials will assess the individual response to each therapeutic option. On the other hand, the increasing insights into the anatomic subtypes of AVMs in recent years has allowed surgeons to approach surgically, with acceptable risks, AVMs located in territories previously considered inoperable.

\section{References}

1. Yasargil MG (1988) Microneurosurgery. IIIA: AVM of the brain, history, embryology, pathological considerations, hemodynamics, diagnostic studies, microsurgical anatomy. Thieme, Stuttgart

2. Du R, Dowd CF, Johnston SC, Young WL, Lawton MT (2005) Interobserver variability in grading of brain arteriovenous malformations using the Spetzler-Martin system. Neurosurgery 57(4):668-675

3. Griessenauer CJ, Miller JH, Agee BS, Fisher WS, Curé JK, Chapman PR, Foreman PM, Fisher WAM, Witcher AC, Walters BC (2014) Observer reliability of arteriovenous malformations grading scales using current imaging modalities. J Neurosurg 120(5):1179-1187

4. Laakso A, Hernesniemi J (2012) Arteriovenous malformations: epidemiology and clinical presentation. Neurosurg Clin N Am 23(1):1-6

5. Davies JM, Kim H, Young WL, Lawton MT (2012) Classification schemes for arteriovenous malformations. Neurosurg Clin N Am 23(1):43-53

6. Tayebi Meybodi A, Lawton MT (2018) Modern classification and outcome predictors of surgery in patients with brain arteriovenous malformations. J Neurosurg Sci 62(4):454-466

7. The arteriovenous Malformation Study Group (1999) Arteriovenous malformations of the brain in adults. N Engl J Med 340(23):1812-1818

8. D'Aliberti G, Talamonti G, Piparo M, Debernardi A, Zella S, Boccardi E, Valvassori L, Nichelatti M (2014) Venous flow rearrangement after treatment of cerebral arteriovenous malformations: a novel approach to evaluate the risks of treatment. World Neurosurg 82(1-2):160-169

9. Luessenhop AJ, Gennarelli TA (1977) Anatomical grading of supratentorial arteriovenous malformations for determining operability. Neurosurgery 1(1):30-35

10. Luessenhop AJ, Rosa L (1984) Cerebral arteriovenous malformations. Indications for and results of surgery, and the role of intravascular techniques. J Neurosurg 60(1):14-22

11. Shi Y-Q, Chen X-C (1986) A proposed scheme for grading intracranial arteriovenous malformations. J Neurosurg 99:484-489

12. Spetzler RF, Martin NA (1986) A proposed grading system for arteriovenous malformations. J Neurosurg 26:476-483

13. de Oliveira E, Tedeschi H, Raso J (1998) Comprehensive management of arteriovenous malformations. Neurol Res 20(8):673-683 
14. Lawton MT, UCSF Brain Arteriovenous Malformation Study Project (2003) Spetzler-Martin grade III arteriovenous malformations: surgical results and a modification of the grading scale. Neurosurgery 52(4):740-749

15. Ponce FA, Spetzler RF (2011) Arteriovenous malformations: classification to cure. Clin Neurosurg 58:10-12

16. Spears J, TerBrugge KG, Moosavian M, Montanera W, Willinsky RA, Wallace MC, Tymianski M (2006) A discriminative prediction model of neurological outcome for patients undergoing surgery of brain arteriovenous malformations. Stroke 37(6):1457-1464

17. Lawton MT, Kim H, McCulloch CE, Mikhak B, Young WL (2010) A supplementary grading scale for selecting patients with brain arteriovenous malformations for surgery. Neurosurgery 66(4):702-713

18. Hafez A, Koroknay-Pál P, Oulasvirta E, Elseoud AA, Lawton MT, Niemelä M, Laakso A (2018) The application of the novel grading scale (Lawton-Young grading system) to predict the outcome of brain arteriovenous malformation. Neurosurgery 84(2):529-536

19. Dumont T, Kan P, Snyder K, Hopkins L, Siddiqui A, Levy E (2015) A proposed grading system for endovascular treatment of cerebral arteriovenous malformations: Buffalo score. Surg Neurol Int 6(1):3

20. Feliciano CE, de Leon-Berra R, Hernandez-Gaitan M, RodriguezMercado R (2010) A proposal for a new arteriovenous malformation grading scale for neuroendovascular procedures and literature review. P R Health Sci J 29:117-120

21. Gupta R, Adeeb N, Moore JM, Motiei-Langroudi R, Griessenauer CJ, Patel AS, Ogilvy CS, Thomas AJ (2016) Validity assessment of grading scales predicting complications from embolization of cerebral arteriovenous malformations. Clin Neurol Neurosurg 151:102-107

22. Lopes DK, Moftakhar R, Straus D, Munich SA, Chaus F, Kaszuba MC (2016) Arteriovenous malformation embocure score: AVMES. J NeuroIntervent Surg 8(7):685-691

23. Pollock BE, Flickinger JC (2002) A proposed radiosurgery-based grading system for arteriovenous malformations. J Neurosurg 89:79-85

24. Robert T, Blanc R, Sylvestre P, Ciccio G, Smajda S, Botta D, Redjem H, Fahed R, Piotin M (2017) A proposed grading system to evaluate the endovascular curability of deep-seated arteriovenous malformations. J Neurol Sci 377:212-218

25. Russell D, Peck T, Ding D, Chen C-J, Taylor DG, Starke RM, Lee C-C, Sheehan JP (2017) Stereotactic radiosurgery alone or combined with embolization for brain arteriovenous malformations: a systematic review and meta-analysis. J Neurosurg 109(3):1338-1348
26. Starke RM, Yen C-P, Ding D, Sheehan JP (2013) A practical grading scale for predicting outcome after radiosurgery for arteriovenous malformations: analysis of 1012 treated patients. J Neurosurg 93(3):981-987

27. Beltramello A, Zampieri P, Ricciardi GK, Pasqualin A, Nicolato A, Sala F, Piovan E, Gerosa M (2008) Operative classification of brain arteriovenous malformations. Interv Neuroradiol 14(1):9-19

28. Beltramello A, Ricciardi GK, Piovan E, Zampieri P, Pasqualin A, Nicolato A, Foroni R, Sala F, Bassi L, Gerosa M (2009) Operative classification of brain arteriovenous malformation. Interv Neuroradiol 15(3):266-274

29. Neidert MC, Lawton MT, Mader M, Seifert B, Valavanis A, Regli L, Bozinov O, Burkhardt J-K (2016) The AVICH score: a novel grading system to predict clinical outcome in arteriovenous malformation-related intracerebral hemorrhage. World Neurosurg 92:292-297

30. Hemphill JC, Bonovich DC, Besmertis L, Manley GT, Johnston SC (2001) The ICH score: a simple, reliable grading scale for intracerebral hemorrhage. Stroke 32(4):891-897

31. Neidert MC, Lawton MT, Kim LJ et al (2018) International multicentre validation of the arteriovenous malformation-related intracerebral haemorrhage (AVICH) score. J Neurol Neurosurg Psychiatry 89(11):1163-1166

32. Sugita K, Takemae T, Kobayashi S (1987) Sylvian fissure arteriovenous malformations. Neurosurgery 21(1):7-14

33. Lawton MT, Lu DC, Young WL (2007) Sylvian fissure arteriovenous malformations. An application of the Sugita classification to 28 surgical patients. Neurosurgery 61:29-38

34. Kim YB, Young WL, Lawton MT (2011) Parafalcine and midline arteriovenous malformations: surgical strategy, techniques and outcomes. J Neurosurg 59(1):984-993

35. Gabarrós Canals A, Rodríguez-Hernández A, Young WL, Lawton MT, Project UBAS (2013) Temporal lobe arteriovenous malformations: anatomical subtypes, surgical strategy and outcomes. J Neurosurg 119(3):616-628

36. Rodríguez-Hernández A, Kim H, Pourmohamad T, Young WL, Lawton MT (2012) Cerebellar arteriovenous malformations. Neurosurgery 71(6):1111-1124

37. Han SJ, Englot DJ, Kim H, Lawton MT (2015) Brainstem arteriovenous malformations: anatomical subtypes, assessment of "occlusion in situ" technique, and microsurgical results. J Neurosurg 58(4):107-117

Open Access This chapter is licensed under the terms of the Creative Commons Attribution 4.0 International License (http://creativecommons. org/licenses/by/4.0/), which permits use, sharing, adaptation, distribution and reproduction in any medium or format, as long as you give appropriate credit to the original author(s) and the source, provide a link to the Creative Commons license and indicate if changes were made.

The images or other third party material in this chapter are included in the chapter's Creative Commons license, unless indicated otherwise in a credit line to the material. If material is not included in the chapter's Creative Commons license and your intended use is not permitted by statutory regulation or exceeds the permitted use, you will need to obtain permission directly from the copyright holder. 